\title{
Carbon nanotube reinforced polymer composites-A state of the art
}

\author{
S BAL* and S S SAMAL ${ }^{\dagger}$ \\ *Department of Physics, ${ }^{\dagger}$ Metallurgical and Materials Engineering Department, National Institute of Technology, \\ Rourkela 769 008, India
}

MS received 26 December 2005

\begin{abstract}
Because of their high mechanical strength, carbon nanotubes (CNTs) are being considered as nanoscale fibres to enhance the performance of polymer composite materials. Novel CNT-based composites have been fabricated using different methods, expecting that the resulting composites would possess enhanced or completely new set of physical properties due to the addition of CNTs. However, the physics of interactions between CNT and its surrounding matrix material in such nano-composites has yet to be elucidated and methods for determining the parameters controlling interfacial characteristics such as interfacial shear stress, is still challenging. An improvement of the physical properties of polymer nanocomposites, based on carbon nanotubes (CNTs), is addicted to a good dispersion and strong interactions between the matrix and the filler.
\end{abstract}

Keywords. Polymer; carbon nanotube; nanocomposite; functionalization; dispersion.

\section{Introduction}

Polymeric nanocomposites (PNCs) or polymer nanostructured materials represent a radical alternative to conventional-filled polymers. The reinforcement of polymers are done by fillers, which play a major role in strengthening the composite. In contrast to the conventional systems where the reinforcement is on the order of microns, discrete constituents on the order of a few nanometers $(\sim 10,000$ times finer than a human hair) exemplify PNCs. Uniform dispersion of these nanoscopically sized filler particles produces ultra-large interfacial area per volume between the nanoelement and host polymer. Thus, new combinations of properties derived from the nanoscale structure of PNCs provide opportunities to circumvent traditional performance trade-offs associated with conventional reinforced plastics, epitomizing the promise of nano-engineered materials. Since the discovery of carbon nanotubes (CNT) by Iijima in 1991 (Walters et al 1999), increasing attention has been paid to this newly emerging material due to its remarkable mechanical and electrical properties (Kracke and Damaschke 2000; Ishikawa et al 2001). There are two main types of carbon nanotubes that can have high structural perfection (Baughman et al 2002). Single walled nanotubes (SWNT) consist of a single graphite sheet seamlessly wrapped into a cylindrical tube (figure 1). Multiwalled nanotubes (MWNT) comprise an array of such nanotubes that are concentrically nested-like rings of a tree trunk (figure 2) (Khare and Bose 2005).

One of the most intriguing applications of CNT is the polymer/CNT nanocomposites (Hersam et al 1998; Laurie

*Author for correspondence (smrutisikha_bal@yahoo.com) et al 1998; Ruiz et al 1998; Fiege et al 1999; Gomes et al 1999; Cai et al 2000). The high mechanical, electrical and thermal properties of CNT makes them ideal candidates as fillers in light weight polymer composite. It is their extraordinary mechanical properties (exceptionally high tensile strength and stiffness) that has aroused particular interest and promoted research into the fabrication of nanotube composite materials (Wong et al 1997). The present paper describes the potential of CNTs as nanofillers in polymers by focusing some light on the preparation and properties of carbon nanotube polymer composites. This review also discusses the special challenges in efficiently exploiting the stability and limitations of the nano-scaled fillers.

\section{CNT based polymer composites}

Polymers and polymer matrix composite materials are being utilized in an increasing number of industrial applications including transportation, automotive, aerospace, defence, sporting goods, energy and infrastructure sectors. This is due to their high durability, high strength, light weight, design and process flexibility, etc. Polymers such as epoxy (Ruiz et al 1998), thermoplastics (Fiege et al 1999), gels (Gomes et al 1999), as well as poly (methyl methacrylate) (PMMA) (Laurie et al 1998) have been used as the matrix. The conductivity, strength, elasticity, toughness, and durability of formed composites may all be substantially improved by the addition of nanotubes. The very high aspect ratio of some carbon nanotubes may also enable them to be aligned with one axis of the composite. This high axial electrical conductivity offers the potential for fabricating conducting polymers. Carbon nanotubes could replace conventional conductive fillers for a range 
of applications like electrostatic discharge (ESD) and electromagnetic interference (EMI) shielding, and a much lower loading of carbon nanotubes can be used to achieve desired conductivity levels (Grimes et al 2000, 2001).

However, the effective utilization of carbon nanotubes in composite applications depends strongly on the ability to homogeneously disperse them throughout the matrix without destroying their integrity. Furthermore, good interfacial bonding is required to achieve load transfer across the CNT-matrix interface, a necessary condition for improving the mechanical properties of the composite (Andrew et al 2002). Load transfer from matrix to CNTs plays a key role in the mechanical properties of composites. If the adhesion between the matrix and the CNTs is not strong enough to sustain high loads, the benefits of the high tensile strength of CNTs are lost. Load transfer depends on the interfacial shear stress between the fibre and the matrix (Schadler et al 1998). A high interfacial shear
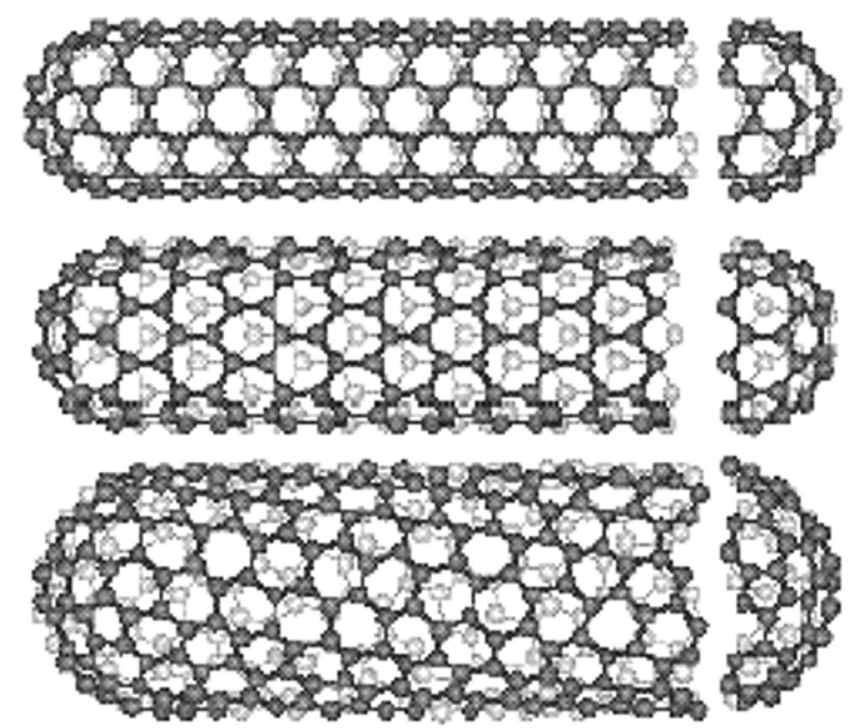

Figure 1. Single wall carbon nanotubes (SWNT).

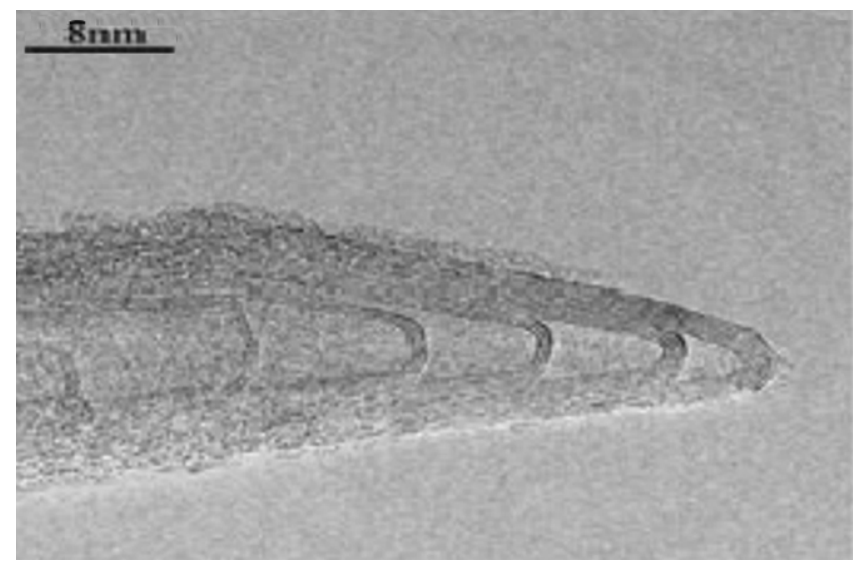

Figure 2. Tip of multi-wall carbon nanotubes (MWNT). stress will transfer the applied load to the fibre over a short distance, and a low interfacial shear stress will require a long distance. There are three main mechanisms of load transfer from a matrix to a filler.

(a) Micro-mechanical interlocking: This could be difficult in nanotube composites due to their atomically smooth surface. Local non-uniformity along a CNT, including varying diameter and bends/kinks at places as a result of non-hexagonal defects, contribute to CNT-polymer adhesion by mechanical interlocking (Wong et al 2003).

(b) Chemical bonding between the nanotubes and the matrix: This improves interfacial interaction through ionic or covalent bond that enables a stress transfer.

(c) Weak van der Waals bonding between the fibre and the matrix: Under no chemical bonding between CNTpolymer, the origins of CNT-polymer interactions are electrostatic and van der Waals forces.

\section{Fabrication and properties of polymer nanocomposites}

Although CNTs have been observed to possess a broad range of extraordinary properties, they are essentially existent at the nanoscale level. Therefore, in order to be able to utilize CNTs and their properties in real-world applications, there must exist certain means or ways that would help us realize the properties of these fascinating nanostructures at macroscopic levels. Significant research work is being done in this area, in an effort to establish standard methods that can accomplish this task of utilizing the properties of CNTs at macroscopic levels. Some of the past work done in this area involved the inclusion of CNTs in a variety of polymers by numerous methods, which resulted in the imparting of some of the properties of nanotubes to the nanocomposites thus formed.

Ajayan et al (1994) reported the first work on a CNTpolymer composite. Purified arc-discharge MWNTs were dispersed in a liquid-epoxy base resin by mechanical stirring. After evacuation to remove trapped air bubbles, heating above $6^{\circ} \mathrm{C}$ for $2 \mathrm{~h}$ hardened pastes of the material. Thin slices were cut and observed by TEM. It was demonstrated that, during cutting, the majority of MWNTs were straightened in the strain direction rather than broken. This result suggested, first, that MWNTs have excellent mechanical properties and second that anisotropy can be induced by a flow in such a material. Only several years later, in 1998, were other works published on CNTpolymer composites.

Schadler et al (1998) reported the dispersion of $5 \mathrm{wt} \%$ MWNTs in an epoxy resin by an ultrasonic treatment. Although the MWNTs were well separated, they remain poorly distributed (figure 3). Mechanical tests and associated Raman spectroscopy revealed that the load transfer to the MWNTs were much higher in compression than in 
tension. It was inferred that, under loading, only the outer layers of the MWNTs are stressed in tension because all the inner tubes are sliding within the outer, whereas all the layers are stressed in compression.

Further investigation on the load transfer in such materials was reported. Both laser-ablation SWNTs and arcdischarge MWNTs were used. It was found (Laurie et al 1998) that the compressive strength of thin- and thickwalled CNTs is more than two orders of magnitude higher than the compressive strength of any known material, which is close to $0.05 \mathrm{GPa}$. Compressive stresses were induced in composite films of $200 \mu \mathrm{m}$ thickness, from the shrinkage of the matrix due to polymerization and by further quenching from room temperature to low temperatures (223$81 \mathrm{~K}$ ) (Laurie and Wagner 1998a; Laurie et al 1999). The obtained values were close to those previously reported for SWNTs and MWNTs, about 5 and 1.8 TPa, respectively (Overney et al 1993a, b; Treacy et al 1996). The fracture of a SWNT-epoxy composite, observed in real time by TEM, revealed a good polymer-CNT wetting and significant CNT-polymer adhesion (Laurie and Wagner 1998b). Raman spectroscopy investigations of SWNTepoxy and MWNT-epoxy composites (Cooper et al 2001) allowed one to determine the effective modulus of the CNTs (about $1 \mathrm{TPa}$ and $0 \cdot 3 \mathrm{MPa}$ for the SWNTs and MWNTs, respectively).

CCVD MWNTs (inner diameter, $5 \mathrm{~nm}$; outer diameter, $10 \mathrm{~nm}$; length, a few micrometers) were dispersed in an epoxy matrix $(0 \cdot 0225-0 \cdot 15 \mathrm{wt} \%$ MWNTs) (Sandler et al 1999). The exposure to ultrasound early in the process and the subsequent intense stirring of the resin dramatically improved the dispersion of MWNTs in the matrix. The percolation threshold was found to be between 0.0225 and $0.04 \mathrm{wt} \%$ MWNTs. An electrical conductivity of about $10^{-2} \mathrm{~S} / \mathrm{m}$, sufficient for antistatic applications, was

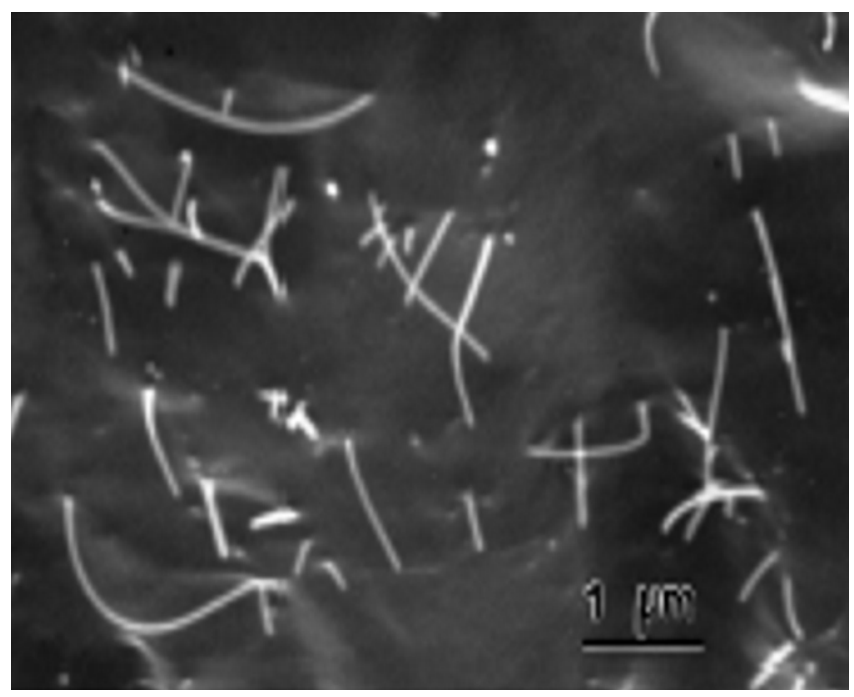

Figure 3. SEM micrograph showing the distribution of nanotubes through the resin. achieved with $0.04 \mathrm{wt} \%$ MWNTs, i.e. using a loading about one-tenth of that needed using carbon black. It is noted that at these low filler fractions, neither the processing behaviour of the matrix nor the surface finish of the samples is adversely affected and that the mechanical properties of the epoxy matrix should not be compromised.

Polyamide/carbon nanotube (PI/CNT) nanocomposites (Cai et al 2004) with different proportions of CNT were fabricated by in situ process. The results showed that the bending strength and microhardness of the PI/CNT nanocomposites increased with increasing CNT content and reached stable values at a certain content of CNT. CNT could effectively enhance the friction-reduction and antiwear capacity of the nanocomposite because it increased the load capacity and mechanical strength of the CNT/PI. The variables such as applied load and sliding speed had a significant influence on friction and wear performance.

The influence of carbon nanotube (CNT) contents on electrical and rheological properties of CNT-reinforced polypropylene (PP) composites was studied. As a result, the volume resistivity of the composites was decreased with (Seo and Park 2004) increasing CNT content and the electrical percolation threshold was formed between 1 and $2 \mathrm{wt} \% \mathrm{CNT}$, which were caused by the formation of conductive chains in the composites (figure 4 ). The viscosity of the composites was increased with the addition of CNT, which was accompanied by an increase in elastic melt properties (figure 5). This could be explained by the higher aspect ratio of $\mathrm{CNT}$ and the composites containing more than $2 \mathrm{wt} \%$ CNT exhibited non-Newtonian curves at low frequency.

Siochia Emilie et al (2004) found that the tensile modulus and yield strength increased with the addition of SWNT loading in a polyamide SWNT composite. The

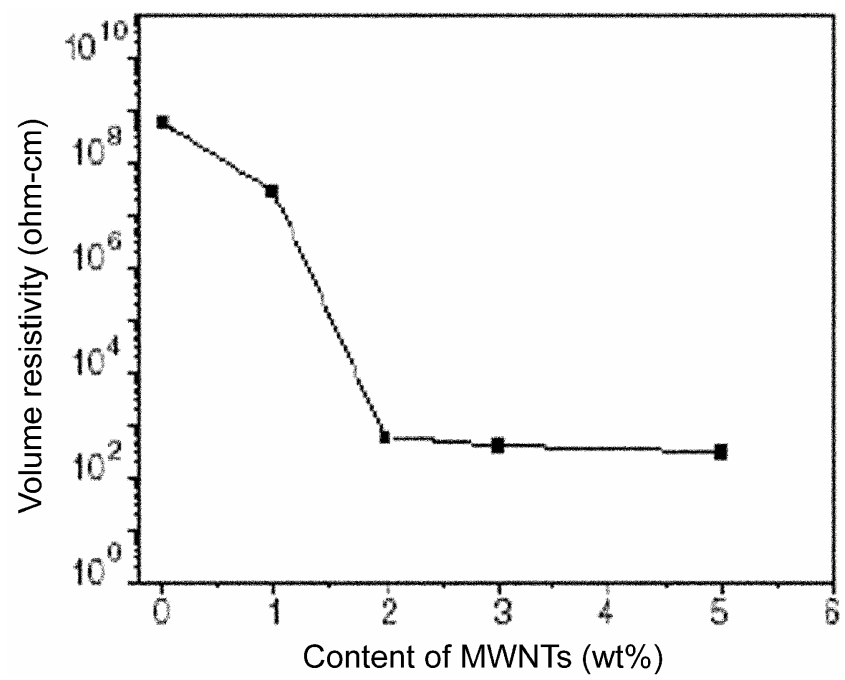

Figure 4. Electrical volume resistivity of MWNT/PP composites as a function of nanotube content. 
increase was much higher than that observed for film samples (which were cast without preferred SWCNT orientation), but much less than what was expected from an oriented discontinuous fibre reinforced polymer composite. This low level of improvement was likely due to inefficient and incomplete dispersion. With the aid of improved dispersion, significant reinforcing effects of the aligned fibres on the mechanical properties are anticipated. Improvements in mechanical reinforcement may also be realized with a matrix designed to promote uniform dispersion by capitalizing on physical interaction with SWCNT inclusions to improve the nanotube/matrix interface so as to maximize load transfer across the interface.

\section{Limitations and challenges}

CNT reinforced polymer composites are seen as a potentially fruitful area for new, tougher or fatigue resistant materials. Although various studies have provided some insights into the nature of $\mathrm{CNT}$-polymer interactions at the interface, the physics of CNT-polymer interactions still await further elucidation, both qualitatively and quantitatively. A relevant question is, will the high modulus and strength predicted for nanotubes be really available when used as fillers? MWNTs are generally entangled in the form of curved agglomerates and SWNTs are produced as bundles. In order to achieve optimal enhancement in the property of the CNTs/polymer composites, there are several key issues to be resolved, i.e. improved dispersion of CNTs, alignment of CNTs in the polymer resin and functionalizations of CNTs surface for good adhesion. A good CNT/ matrix interfacial bonding and a perceptible reinforcement of the matrix with the nanotubes can bring improvement to the fracture strength of the composite by ensuring a shear stress transfer to the reinforcement.

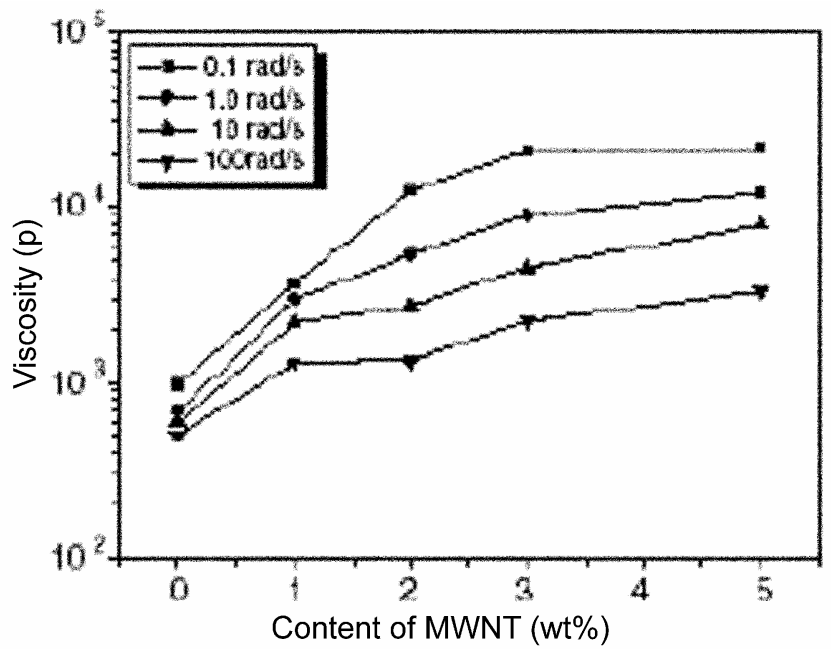

Figure 5. Viscosity of MWNT/PP composites as a function of CNT content.

\subsection{Influence of functionalization on polymer-CNT composites}

The effect of functionalization to achieve bonding between the matrix and the nanotubes was shown by Frankland et al (2002) via molecular dynamics simulation. They have shown that an improvement in mechanical properties can already be achieved if less than $1 \%$ of the carbon atoms of the carbon nanotubes form reactive bridges to the matrix. A breakthrough in the development of carbon nanotube-reinforced epoxies could be made via chemical functionalization of CNTs with multifunctional amines. The improvement of the bonding to the matrix as well as a better dispersion of the CNTs could be observed (Zhu et al 2003, 2004). A schematic representation of functionalization process is shown in figure 6 (Schulte et al 2005). In the first step, an oxidative treatment of the nanotubes was used to develop carboxylic groups. This leads to an opening of the CNT cap (figure 7), which would enable a direct

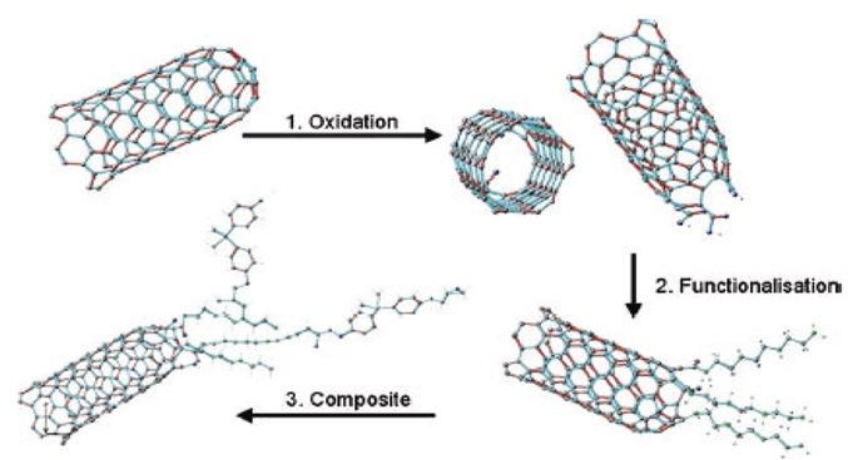

Figure 6. Functionalization process of CNTs showing the steps from the oxidation to the composite manufacturing.

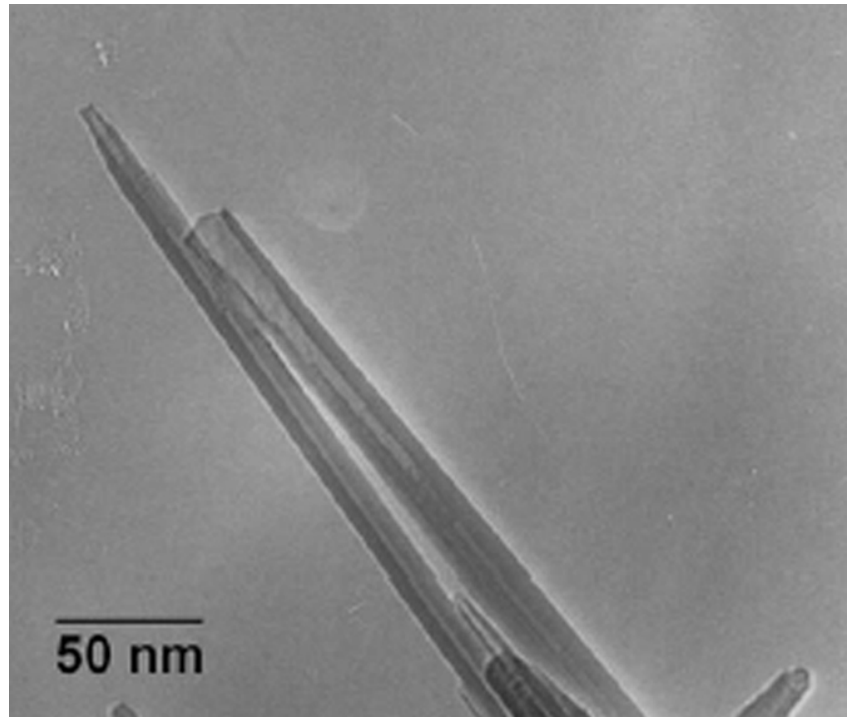

Figure 7. Opening of the caps of oxidized arc grown MWCNTs. 
bonding of the tube ends to the matrix via the carboxylic groups. In the second step, the carboxylic groups would react with multifunctional amines and form bonds (either ionic or under the given conditions, covalent) to these amines via an acid-base reaction. In the third step, with the addition of the epoxy resin, the free amino functions on the surface of CNTs will react with the epoxy molecules forming equivalent bonds, which lead to an improved nanotube matrix bonding.

Shaffer and coworkers have demonstrated that acid treatment enables stable aqueous solution of catalytically produced MWNTs to be prepared (Shaffer et al 1998). Solution based methods have also been used to produce nano tube/polystyrene composites. Both SWNTs and MWNTs were solubilized by functionalizing with a polystyrene copolymer (Hill et al 2002). This was achieved by first acid treating the tubes and then carrying out esterification of the surface bound carboxylic acid. The polymer modified carbon nanotubes were shown to be soluble in common organic solvent. To prepare composites, polystyrene was dissolved in the nanotube solution and nanotube polystyrene thin films prepared using wet casting.

Another common method to assimilate the polarity of CNT and matrix, is the use of surfactants. The advantage of this procedure is the physical adhesion, which does not reduce the structural quality of CNTs, whereas a covalent integration of functional groups is always related to structural changes of graphitic layers. Similar to surfactants, conjugated polymers (e.g. PmPV) can also physically bond to CNTs and be utilized to improve the compatibility of CNTs and matrix (McCarthy et al 2000). Influence of a non-ionic surfactant (Tertigol NP 7) in the nanocomposite processing has been analysed by Cui et al (2003). These results demonstrated that the glass transition temperature is increasing with the nanotube content, and on the other hand, the percolation threshold is found for a rather high critical volumic concentration.

\subsection{Fundamental aspects of dispersion}

Functionalization of nanotube is not always necessary to prepare a polymer composite. A good dispersion not only makes more filler surface area available, but also prevents aggregation of the filler action as stress concentrators as well as slippage of nanotube during composites loading, which all decrease the performance of the composites greatly (Liu and Wagner 2005). Since nanofiller has an extremely high surface area, only a few polymer molecules can penetrate between them while covered with matrix. Now, if the CNTs should evenly be distributed in the matrix polymer, some challenges have to be faced like length of the tubes, their entanglement, volume fraction and the high matrix viscosity. With increasing CNT length, the resulting interactive forces similar to the influence of the molecular weight (chain length) hinders the separation of the tubes. The shear forces introduced into the suspension in order to separate the agglomerates and to disperse the individual CNTs is controlled by the manufacturing process. Dispersion of the nanotubes can be done through various methods like sonication, stirring, calendaring etc.

Sonication method is only suitable for very low viscous matrix materials and small volumes because ultrasonic devices have a high impact of energy, but introduce low portion of shear forces. Again the effectiveness of the dispersion is limited as the local introduction of the energy leads to a rupture and damage of CNTs reducing the overall aspect ratio ( $\mathrm{Lu}$ et al 1996). In figure 8, MWCNTs were sonicated for $20 \mathrm{~min}$ at $30 \%$ amplitude, mixed with the epoxy resin and again sonicated for $10 \mathrm{~min}$. After that, agglomerates still remained.

A proper way to apply the sonication technique to produce CNT-nanocomposites is to disperse them in an appropriate solvent (i.e. acetone, ethanol etc), which first allows the agglomerates to be separated due to vibrational energy. The suspension can later be mixed with the polymer and the solvent can simply be evaporated by heating. Morphology and physical properties of poorly dispersed (without solvent) and well-dispersed (in ethanol) CNTs/epoxy composites have been analysed (Song and Youn 2004). Figure 9 shows the FESEM images of the composites indicating the remaining portions of agglomerates in the poorly dispersed one. They reduce reinforcing effects of CNTs and result in a lower tensile strength (figure 10), electrical conductivity (figure 11) and thermal conductivity (figure 12) in comparison to well-dispersed (with solvent) CNTs/epoxy composite. A reduction in the agglomerate size can be achieved with a functionalization process (Gojny et al 2003). A combination of sonication and an oxidative process where the functional groups, which develop on the surfaces of the CNTs, lead to steric hindering and electrostatic interactions with the solvent, resulting in a better distribution of CNTs in the matrix.

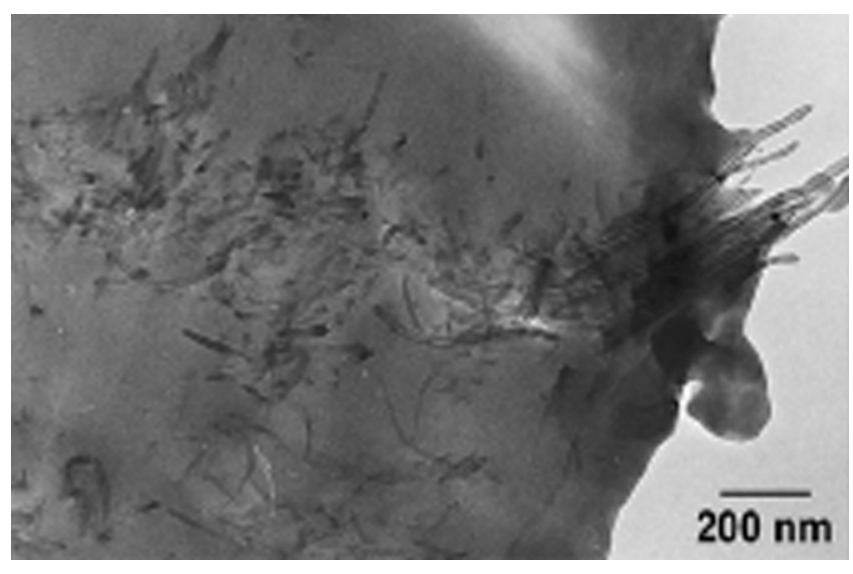

Figure 8. TEM micrograph of sonicated MWCNT/epoxy composites. 

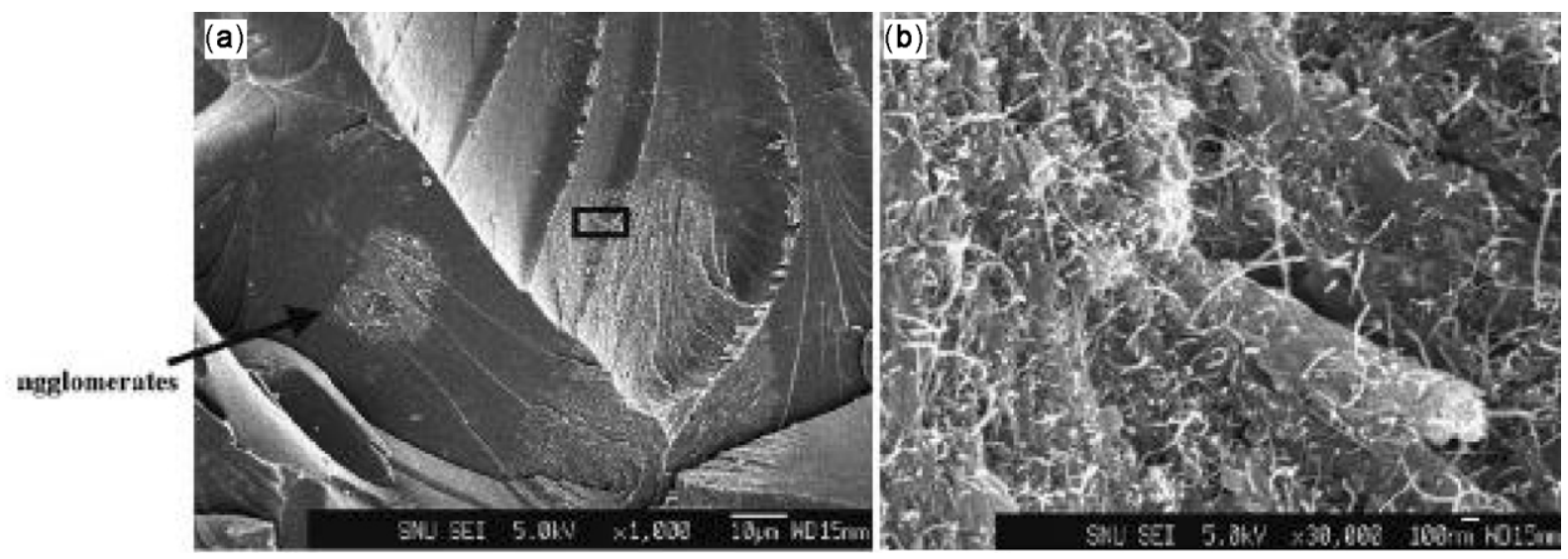

Figure 9. FESEM images of CNTs/epoxy nanocomposites prepared without using the solvent ((a) \& (b)).

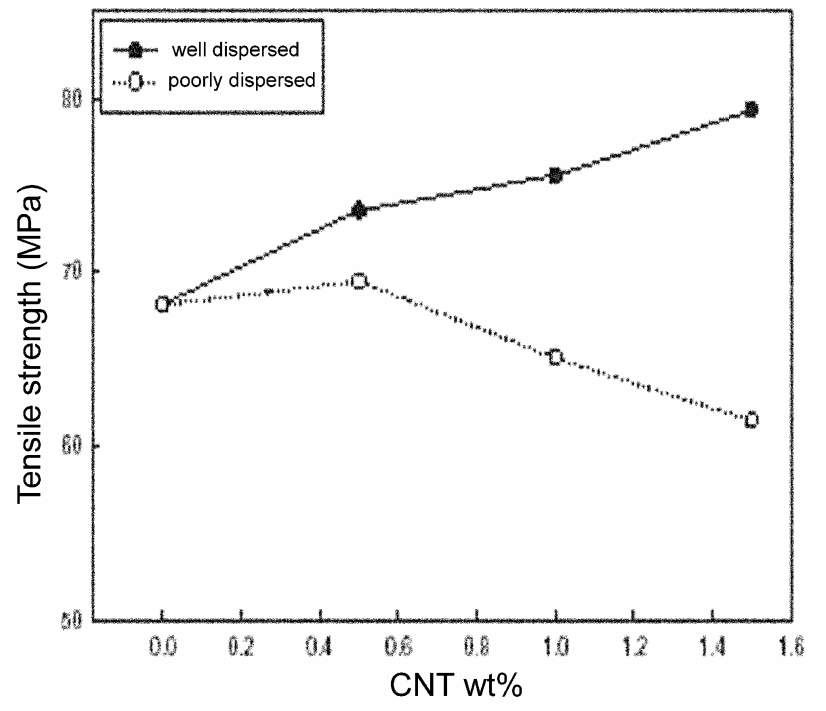

Figure 10. Tensile strength of CNT epoxy composite with respect to CNT loading.

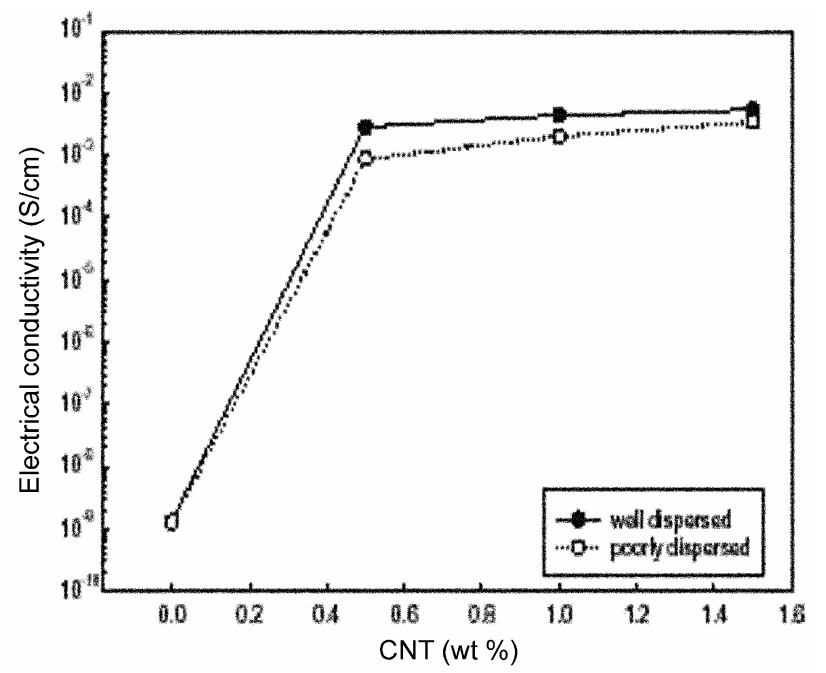

Figure 11. Electrical conductivity of nanocomposites with different degrees of CNTs dispersion as a function of carbon content.

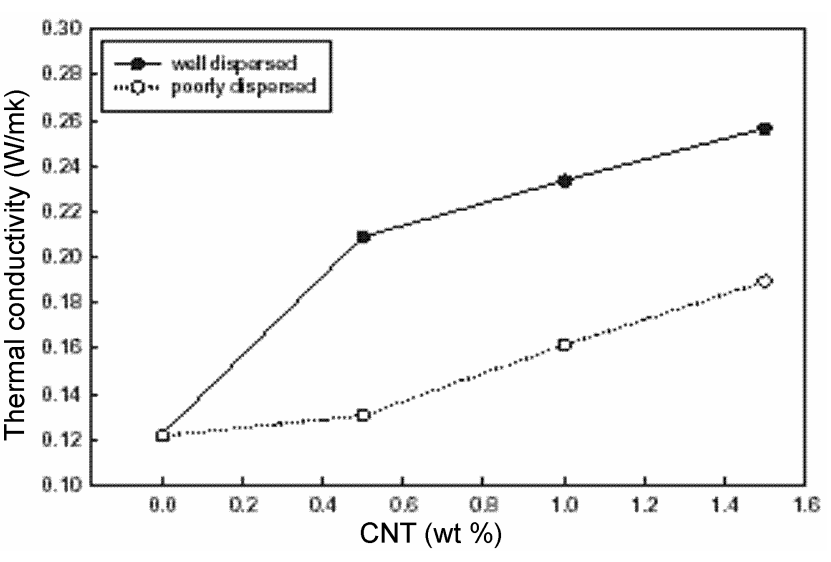

Figure 12. Thermal conductivity of the nanocomposites with respect to carbon loading.

Stirring is a common technique to disperse nanoparticles. The dispersion result can be controlled by size and shape of the propeller and the mixing speed. After intensive stirring of MWCNTs in epoxy resin a relative fine dispersion can be achieved (Sandler et al 1999). However, the re-agglomeration of MWCNTs is mainly caused by frictional contacts and elastic interlocking mechanisms (Schmid and Klingenberg 2000).

The application of a mini-calender to disperse carbon nanotubes and nano-particles, in general, became a very promising approach to reach a good state of dispersion (Gojny et al 2004). A major advantage of this method is, besides the improved dispersion results, efficient manufacturing of larger amounts of nano-composites can be achieved. Typical micrographs showing dispersion of MWNTs after shear mixing (figure 13) was based upon the uniformity of nanotube distribution across the specimen and frequency of occurrence of agglomerates. Alternatively, inadequate dispersion may be represented by discontinuities at the boundaries between mixing zones containing different concentrations of dispersed MWNTs (Andrew et al 2002). High shear mixing to disperse 

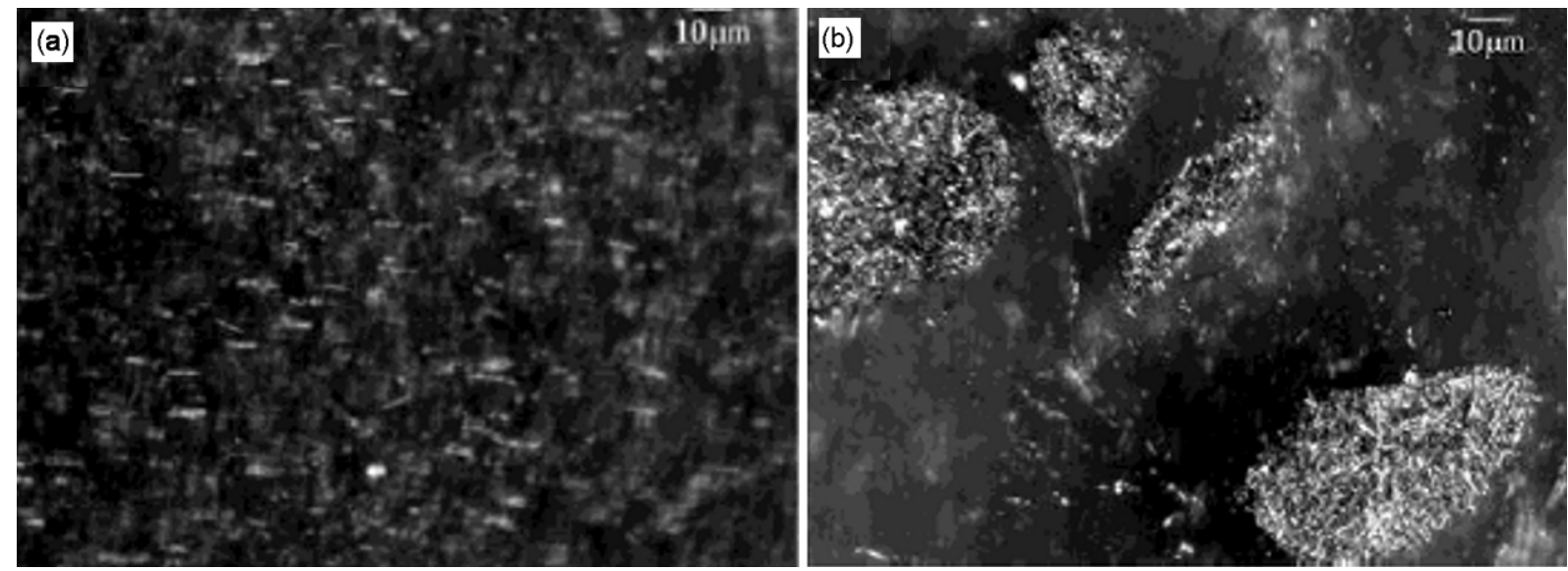

Figure 13. Optical micrographs showing (a) well (uniform) dispersion and (b) poor dispersion, corresponding to high mixing energy and low mixing energy input into the shear mixing process.

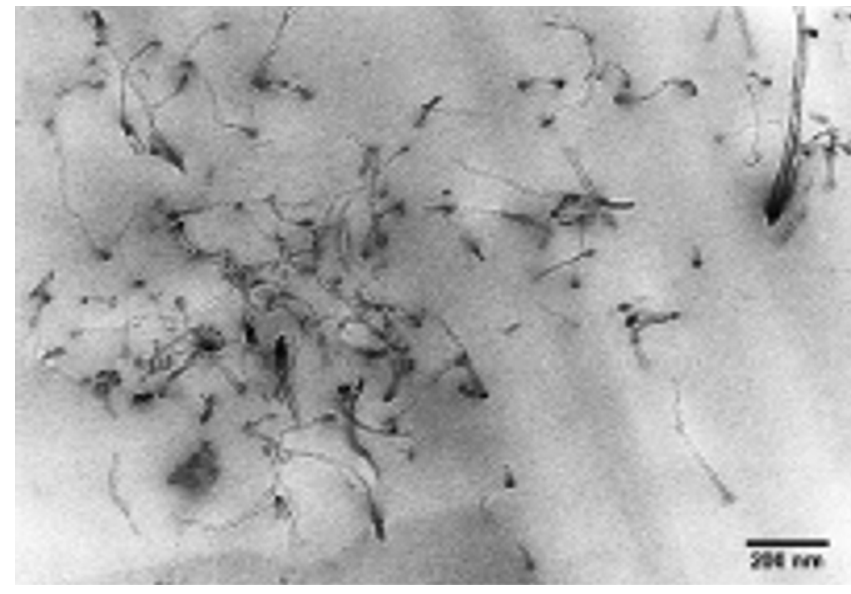

Figure 14. TEM micrographs of calendaring of MWCNT/ epoxy.

MWNTs may result in tube breakage. The results show that some breakage does occur and that means tube length decreases with increasing energy input. The rate at which mean tube length is reduced diminishes as the material is dispersed and tube separation increases. Hence, tube breakage is not a serious problem and the aspect ratio of the tubes remains very high, reducing from 1000-250. Good dispersion can, therefore, be achieved at the expense of an acceptable reduction in tube length. From figure 14, high quality of dispersion can be seen. Only a few small aggregates remain, which are smeared out and well penetrated by the epoxy matrix.

\section{Conclusions}

Nano-particles, in general, and carbon nanotubes, in particular, are desirable particles to modify material properties of polymers. In order to disperse CNTs in the polymer homogeneously, the entanglement of CNTs produced by the synthesis and agglomerates of CNTs caused by the intermolecular van der Waals force must be broken for homogenization that will make more filler surface area available. A further enhancement of the compatibility to the composite material could be achieved by a chemical functionalization of the carbon nanotube surface, through covalent or ionic bonds to the polymeric matrix. These bonds enable a stress transfer between the polymer and CNTs, which leads to improved interfacial interactions. Again the covalent bonding is stronger as physical interactions and simulations predict a negligible influence on the mechanical performance of CNTs. Therefore, the chemical surface functionalization of CNTs along with perceptible dispersion of nanotubes in the matrix are the key issues in developing CNT/polymer composites.

\section{Future scope}

For nearly a decade of research, many studies have been conducted and it has been expected that CNTs could play a role as an excellent reinforcement in the polymer composite that has not yet been fully realized (Schadler et al 1998; Allaoui et al 2002; Wong et al 2003). Such studies were of value in understanding the reasons for composite failure and in identifying the critical issues, which need to be addressed. The factors like intratube load transfer in MWNTs and shearing in SWNT bundles are the central issues for the production of composites with exceptional mechanical properties. In spite of the obstacles in the development of nanotube-based composites, the good load transfer measured between a matrix and CNTs is very encouraging for the future. Now that these problems have been identified, we believe that solutions will be found for making CNT polymer composites in which the exceptional Young's modulus and strength of CNTs will be reflected in the overall physical properties. 


\section{References}

Ajayan P M, Stephan O, Colliex C and Trauth D 1994 Science 2651212

Allaoui A, Bai S, Cheng H M and Bai J B 2002 Composite Sci. \& Technol. 621993

Andrew Rodney, Jacques David, Qian Dali and Rantell Terry 2002 Acc. Chem. Res. 351008

Baughman Ray H, Zakhidov Anvar A and Heer Walt A de 2002 Science 297787

Cai H, Yan Fengyuan and Xue Qunji 2004 Mater. Sci. \& Engg. A364 94

Cai L, Tabata H and Kawai T 2000 Appl. Phys. Lett. 773105

Cooper C A, Young R J and Halsall M 2001 Composites A32 401

Cui S, Canet R, Derre A, Couzi M and Delhaes P 2003 Carbon 41797

Fiege G B M, Altes A, Heiderhoff R and Balk L J 1999 J. Phys. D32 L13

Frankland S J V, Caglar A, Brenner D W and Griebel M $2002 \mathrm{~J}$. Phys. Chem. B106 3046

Gojny F H, Nastalczyk J, Roslanic Z and Schulte K 2003 Chem. Phys. Lett. 370820

Gojny F H, Wichmann M H G, Köpke U, Fiedler B and Schulte K 2004 Composites Sci. Technol. 642363

Gomes S, Trannoy N and Grossel P 1999 Meas. Sci. Technol. 10805

Grimes C A, Mungle C, Kouzoudis D, Fang S and Eklund P C 2000 Chem. Phys. Lett. 319460

Grimes C A, Dickey E C, Mungle C, Ong K G and Qian D 2001 J. Appl. Phys. 904134

Hersam M C, Hoole A C F, O'Shea S J and Welland M E 1998 Appl. Phys. Lett. 72915

Hill D E, Lin Y, Rao A M, Allard L F and Sun Y P 2002 Macromolecules 359466

Ishikawa H, Fudetani S and Hirohashi M 2001 Appl. Surf. Sci. 17856

Khare Rupesh and Bose Suryasarathi 2005 J. Miner. \& Mater. Charact. \& Eng. 431

Kracke B and Damaschke B 2000 Appl. Phys. Lett. 77361

Laurie O and Wagner H D 1998a J. Mater. Res. 132418

Laurie O and Wagner H D 1998b Appl. Phys. Lett. 733527
Laurie O, Cox D E and Wagner H D 1998 Appl. Phys. Lett. 81 1638

Laurie O, Wagner H D, Zhang Y and Lijima S $1999 A d v$. Mater. 11931

Liu Luqi and Wagner H D 2005 Composites Sci. \& Technol. 65 1861

Lu K L, Lago M, Chen Y K, Green M L H, Harris P J F and Tsang S C 1996 Carbon 34814

McCarthy B, Coleman J N, Curran S A, Dalton A B, Davey A P and Konya Z 2000 J. Mater. Sci. Lett. 192239

Overney G, Zhong W and Tomanek D Z 1993a Z. Phys. D27 93

Overney G, Zhong W and Tomanek D Z 1993b Phys. Lett. 370 820

Ruiz Facundo, Sun W D, Pollak Fred H and Venkatraman Chandra 1998 Appl. Phys. Lett. 731802

Sandler J K W, Shaffer M S P, Prasse T, Bauhofer W, Schulten $\mathrm{K}$ and Windle A H 1999 Polymer 405967

Schadler L S, Giannaris S C and Ajayan P M 1998 Appl. Phys. Lett. 733842

Schmid C F and Klingenberg D J 2000 Phys. Rev. Lett. 84290

Schulte K, Gojny F H, Fiedler B, Sandler J and Bauhofer W 2005 Carbon nanotube reinforced polymers. A state of the art-review, in Polymer-composites-from nano to macro scale (eds) K Friedrich et al pp 3-23

Seo Min-Kang and Park Soo-Jin 2004 Chem. Phys. Lett. 39544

Shaffer M S P, Fan X and Windle A H 1998 Carbon 361603

Siochia Emilie J, Working Dennis C, Park Cheol, Lilleheia Peter T, Rouse Jason H, Topping Crystal C, Bhattacharyya A R and Kumar Satish 2004 Composites B35 439

Song Young Seok and Youn Jae Ryoun 2004 e-Polymers No. 0801

Treacy M M J, Ebbesen T W and Gibson J M 1996 Nature (London) 381678

Walters D A, Ericson L M, Casavant M J, Liu J, Colbert D T, Smith K A and Smalley R E 1999 Appl. Phys. Lett. 743803

Wong E W, Sheehan P E and Lieber C M 1997 Science 2771971

Wong M, Paramsothy M, Xu X J, Ren Y, Li S and Liao K 2003 Polymer 447757

Zhu J, Kim J D, Peng H, Margrave J L, Khabashesku V N and Barrera E V 2003 Nanoletters 31107

Zhu J, Peng H, Rodriguez-Macias F, Margrave J L, Khabashesku V N and Imam A M 2004 Adv. Funct. Mater. 14643 